\title{
A Wideband and Polarization-Independent Metasurface Based on Phase Optimization for Monostatic and Bistatic Radar Cross Section Reduction
}

\author{
Jianxun Su, ${ }^{1}$ Yao Lu, ${ }^{1}$ Zengrui Li, ${ }^{1}$ Rongrong Zhang, ${ }^{2}$ and Yaoqing (Lamar) Yang ${ }^{3}$ \\ ${ }^{1}$ Electromagnetic Laboratory, Communication University of China, Beijing 100024, China \\ ${ }^{2}$ CommScope Telecommunications China Co. Ltd., Suzhou 215021, China \\ ${ }^{3}$ Department of Electronic and Computer Engineering, University of Nebraska-Lincoln, Lincoln, NE 68182, USA
}

Correspondence should be addressed to Zengrui Li; zrli@cuc.edu.cn

Received 15 May 2016; Revised 27 July 2016; Accepted 7 August 2016

Academic Editor: Shah N. Burokur

Copyright (C) 2016 Jianxun Su et al. This is an open access article distributed under the Creative Commons Attribution License, which permits unrestricted use, distribution, and reproduction in any medium, provided the original work is properly cited.

\begin{abstract}
A broadband and polarization-independent metasurface is analyzed and designed for both monostatic and bistatic radar cross section (RCS) reduction in this paper. Metasurfaces are composed of two types of electromagnetic band-gap (EBG) lattice, which is a subarray with " 0 " or " $\pi$ " phase responses, arranged in periodic and aperiodic fashions. A new mechanism is proposed for manipulating electromagnetic (EM) scattering and realizing the best reduction of monostatic and bistatic RCS by redirecting EM energy to more directions through controlling the wavefront of EM wave reflected from the metasurface. Scattering characteristics of two kinds of metasurfaces, periodic arrangement and optimized phase layout, are studied in detail. Optimizing phase layout through particle swarm optimization (PSO) together with far field pattern prediction can produce a lot of scattering lobes, leading to a great reduction of bistatic RCS. For the designed metasurface based on optimal phase layout, a bandwidth of more than $80 \%$ is achieved at the normal incidence for the $-9.5 \mathrm{~dB}$ RCS reduction for both monostatic and bistatic. Bistatic RCS reduction at frequency points with exactly $180^{\circ}$ phase difference reaches $17.6 \mathrm{~dB}$. Both TE and TM polarizations for oblique incidence are considered. The measured results are in good agreement with the corresponding simulations.
\end{abstract}

\section{Introduction}

The reflection phase control for EM wave impinging on a surface is important in both fundamental EM researches and device technologies. Many phenomena and applications are related to the reflection phase control, such as EM wave absorber [1], high impedance surface and artificial magnetic conductor (AMC) $[2,3]$, reflectarray antennas [4], and beam forming and beam scanning antennas.

Reshaping the scattering pattern can effectively suppress backward reflection. Scattering energy is redirected to other directions. In 2007, Paquay et al. [5] proposed a planar structure for RCS reduction, based on a combination of artificial magnetic conductors (AMC) and perfect electric conductors (PEC) in a chessboard-like configuration. The backscattering field can be effectively canceled by redirecting it along other angles. However, the narrow in-phase reflection bandwidth of the AMC restricts the RCS reduction frequency range. In [6], a planar monolayer chessboard structure is presented for broadband radar cross section reduction using AMC technology. More than $55 \%$ frequency bandwidth with a monostatic RCS reduction larger than $10 \mathrm{~dB}$ is obtained and four scattered beams are produced. In another similar work [7], two-dimensional phase gradient metasurfaces of a chessboard-like configuration have been proposed for wideband RCS reduction. The $-5 \mathrm{~dB}$ RCS reduction bandwidth of $66 \%$ is achieved for TE and TM polarizations at the normal incidence. For those rectangular checkerboard surfaces with periodic arrangement, four scattering beams are produced and bistatic RCS reduction is about $8.1 \mathrm{~dB}$. In 2015, Chen et al. [8] proposed a hexagonal checkerboard surface of periodic fashion, with the $-10 \mathrm{~dB}$ monostatic RCS reduction bandwidth of about $61 \%$, which can create six bistatic RCS lobes, leading to further bistatic RCS reduction (8.9 dB). In [9] Yang 
et al. proposed a metasurface with nonuniform phase distribution for suppression of specular reflections, with the $-7 \mathrm{~dB}$ RCS reduction bandwidth of about $42 \%$ for $y$-polarized and $x$-polarized incident waves. In addition, metamaterial absorber has been proposed as another way to achieve RCS reduction $[10,11]$. In 2013, Singh et al. proposed a broadband millimeter wave metamaterial absorber based on embedding of dual resonators [12], with an absorber at $77 \mathrm{GHz}$ with a bandwidth of $8 \mathrm{GHz}$ and peak absorption of greater than $98 \%$.

Metasurfaces, a new class of metamaterials that consist of only a monolayer of planar metallic structures, have shown great promise for achieving full control of the reflected wavefront with low fabrication cost [13]. Metasurfaces are capable of generating abrupt interfacial phase changes and provide a unique way to control the local wave front at the subwavelength scale [14].

In this paper, an ultrathin nonabsorptive metasurface is designed for broadband RCS reduction. A new mechanism is proposed for manipulating EM scattering and realizing monostatic and bistatic RCSs reduction through optimizing reflection phase. The main contributions of this paper are as follows.

(1) Two unit cells, square ring patch and small circular patch, are optimized to achieve $86.8 \%$ bandwidth for about $180^{\circ}\left( \pm 37^{\circ}\right)$ reflection phase difference.

(2) Theoretical analysis of far field RCS pattern for the metasurface is derived based on phase-only planar array synthesis.

(3) Optimizing the reflection phase layout of the metasurface using array pattern synthesis (APS) and particle swarm optimization (PSO) can create a lot of bistatic RCS lobes ( $\gg 4$ or 6 ), leading to a great reduction of bistatic RCS.

(4) The number of bistatic RCS lobes increases as the lattice number increases. Thus, as the size of metasurface increases, the reduced magnitude of monostatic and bistatic RCSs becomes larger.

(5) The designed metasurface is polarization-independent.

(6) Specular reflection and bistatic RCS for oblique incidence with TE and TM polarization can also be effectively suppressed.

\section{EBG Unit Cell Design}

The variation of reflection coefficient phase is one of the unique properties of EBG structure, because the phase of the reflected field changes continuously from $180^{\circ}$ to $-180^{\circ}$ versus frequency while, ideally, the magnitude of the reflection coefficient of a lossless ground plane is unity. We consider the metasurface consists of only two EBG structures under the normal incidence of a plane wave.

For a scatterer, scattered power is proportional to the incident power and its $\operatorname{RCS}\left(\left|E^{s}\right|^{2} \sim \sigma\left|E^{0}\right|^{2}\right)$. Relative to a perfectly electrical conductor (PEC) plate with the same size, the RCS reduction of the metasurface can be expressed as

$$
\begin{aligned}
\text { RCS reduction } & =10 \log \frac{\sigma_{\text {metasurface }}}{\sigma_{\mathrm{PEC}}} \\
& =10 \log \frac{\left|\vec{E}_{\text {metasurface }}^{S}\right|^{2}}{\left|\vec{E}_{\mathrm{PEC}}^{S}\right|^{2}} \\
& =10 \log \frac{\left|\vec{E}^{0} e^{j \varphi_{1}}+\vec{E}^{0} e^{j \varphi_{2}}\right|^{2}}{4\left|\vec{E}^{0} e^{j \varphi}\right|^{2}} \\
& =10 \log \frac{1+\cos \left(\varphi_{1}-\varphi_{2}\right)}{2} .
\end{aligned}
$$

In order to achieve $10 \mathrm{~dB}$ RCS reduction, reflection phase difference between two EBG structures needs to satisfy

$$
143^{\circ} \leq|\Delta \varphi|=\left|\varphi_{1}-\varphi_{2}\right| \leq 217^{\circ} .
$$

Here, Rogers RT/duroid 5880 material with thickness $h=62 \mathrm{mil}$ and dielectric constant $\varepsilon_{r}=2.2$ (loss tangent $\tan \delta=0.0009$ ) has been chosen as substrate for the ultrathin metasurface. Square ring patch and small circular patch were chosen as the EBG unit cell. The reflection phase of small circular patch changes approximately linearly with frequency. For square ring patch, when the frequency is fixed, the reflection phase change with side length is relatively larger. Thus the dimensions of both unit cells can be easily optimized to achieve about $180^{\circ}$ reflection phase difference in the largest possible frequency band. Two EBG unit cells are simulated by method of moments (MoM) based Ansys Designer using periodic boundary condition (PBC). This optimization process results in the dimensions depicted in Figure 1. The phases of the two EBG structures, as well as the phase difference between them, are plotted in Figure 2. The reflection phase of EBG structure reaches $0^{\circ}$ at the resonant frequencies, which are $14.7 \mathrm{GHz}$ and $32.1 \mathrm{GHz}$ for square ring patch and small circular patch, respectively. In particular, from $15.2 \mathrm{GHz}$ to $38.5 \mathrm{GHz}(86.8 \%$ bandwidth), the phase difference ranges from $143^{\circ}$ to $217^{\circ}$ and is exactly $180^{\circ}$ at $16.9 \mathrm{GHz}$ and $31.1 \mathrm{GHz}$. Therefore, a broadband behavior of the proposed metasurface is expected. Here, the square ring patch is considered as " 0 " element; then small circular patch is " $\pi$ " element.

\section{Far Field Pattern Prediction}

Consider a metasurface under normal incidence of plane wave, which consists of a two-dimensional array of $M \times N$ EBG lattices. The lattices are uniformly spaced with $d_{x}$ in $x$ 


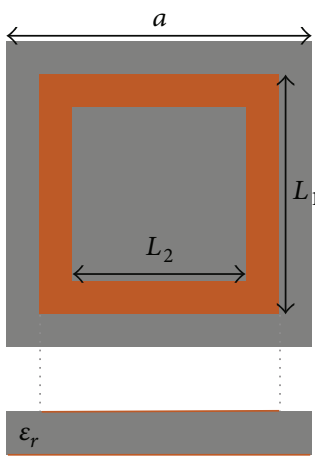

(a)

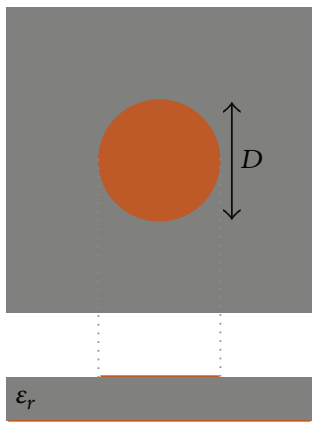

(b)

FIgURe 1: Two unit cells: (a) square ring patch and (b) small circular patch. Dimensions are in mm: $a=2.6, D=1, L_{1}=1.6$, and $L_{2}=2.4$.

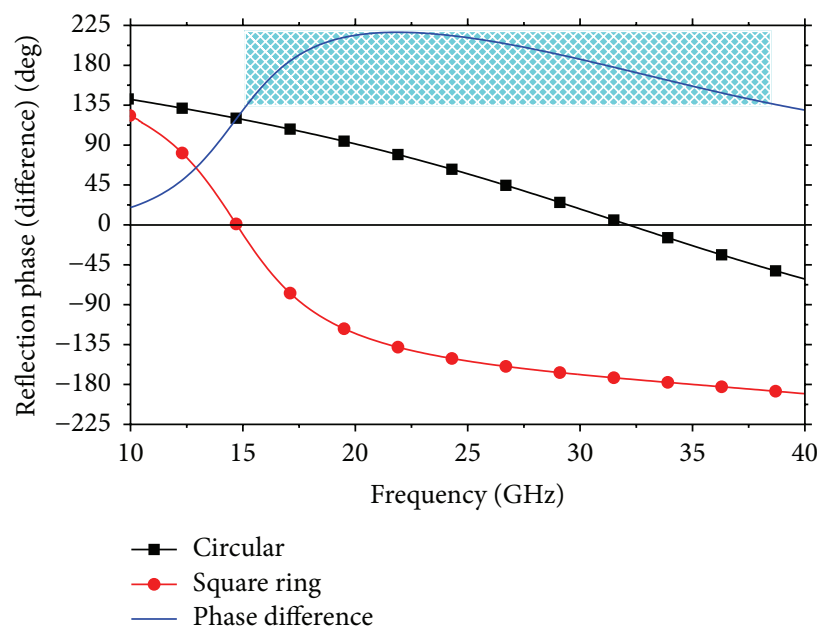

FIGURE 2: Reflection phases of two EBG structures and phase difference between them.

direction and $d_{y}$ in $y$ direction. The total $2 \mathrm{D}$ array pattern can be expressed as [15]

$$
\begin{aligned}
& F(\theta, \varphi)=\mathrm{EP}(\theta) \cdot \operatorname{AF}(\theta, \varphi), \\
& \operatorname{EP}(\theta)=\cos ^{\mathrm{EF} / 2} \theta, \\
& \operatorname{AF}(\theta, \varphi) \\
& \quad=\sum_{m=1}^{M} \sum_{n=1}^{N} e^{-j\left[(2 \pi / \lambda) \sin \theta\left(\cos \varphi \cdot m d_{x}+\sin \varphi \cdot n d_{y}\right)+\emptyset(m, n)\right],}
\end{aligned}
$$

where EP and AF are the element pattern and array factor, respectively. $\emptyset(m, n)=\emptyset_{x}(m)+\emptyset_{y}(n)$ is the initial phase of the element; $\theta$ and $\varphi$ are the elevation and azimuth angles of an arbitrary direction, respectively. A good expression for modeling the element pattern is a cosine function raised to a power that is called the element factor (EF).
Array factor can be separated as

$$
\begin{aligned}
\operatorname{AF}(\theta, \varphi)= & \operatorname{AF}_{U}(\theta, \varphi) \cdot \operatorname{AF}_{V}(\theta, \varphi) \\
= & \sum_{m=1}^{M} e^{-j\left[2 \pi m d_{x} U / \lambda+\emptyset_{x}(m)\right]} \\
& \cdot \sum_{n=1}^{N} e^{-j\left[2 \pi n d_{y} V / \lambda+\emptyset_{y}(n)\right]}
\end{aligned}
$$

where $U=\sin \theta \cos \varphi$ and $V=\sin \theta \sin \varphi \cdot \mathrm{AF}_{U}$ and $\mathrm{AF}_{V}$ are periodic functions with the periods $P_{U}=\lambda / d_{x}$ and $P_{V}=$ $\lambda / d_{y}$, respectively.

The equation for the directive gain is

$$
D(\theta, \varphi)=\frac{4 \pi F^{2}(\theta, \varphi)}{\int_{\varphi=0}^{2 \pi} \int_{\theta=0}^{\pi} F^{2}(\theta, \varphi) \sin \theta d \theta d \varphi} .
$$

Suppose the impinging plane wave has a power density $w_{i}$; then the intercepted EM energy by metasurface is $A w_{i}$. $A=M N d_{x} d_{y}$ is the aperture area [16]. The scattered power density is

$$
w_{s}(\theta, \varphi)=\frac{A w_{i} D(\theta, \varphi)}{4 \pi R^{2}},
$$

where $R$ is the far field distance. Therefore, the far field RCS pattern of the metasurface can be calculated by

$$
\sigma(\theta, \varphi)=A \cdot D(\theta, \varphi)
$$

In this paper, the maximum RCS was defined as follows:

$$
\text { maximum } \mathrm{RCS}=\max _{\theta, \varphi}[\sigma(\theta, \varphi)] .
$$

Maximum RCS is utilized to evaluate the suppression degree of bistatic RCS.

\section{Periodic Arrangement}

Nonabsorptive RCS reduction of specular surface can be achieved by cancellation of the reflected field. This can be 


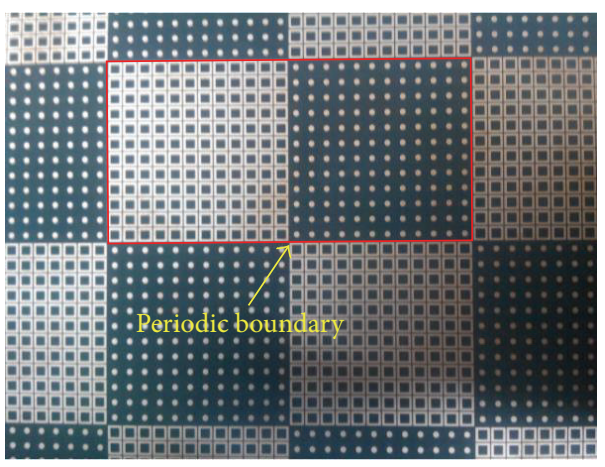

(a)

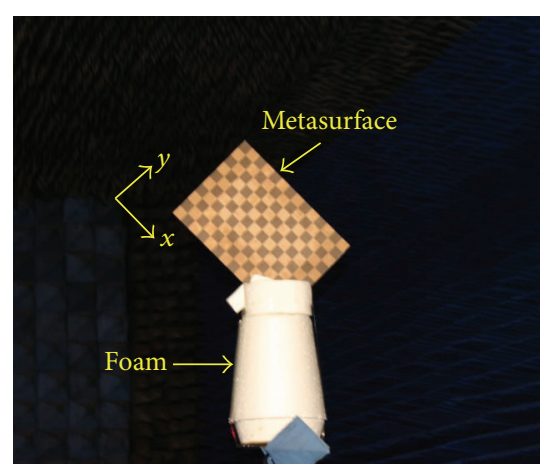

(b)

FIGURE 3: (a) Manufactured metasurface with periodic arrangement. (b) RCS measurement inside an anechoic chamber.

accomplished by covering the object by the metasurface which consisted of " 0 " and " $\pi$ " lattices in a periodic fashion [5-8]. A general rectangular metasurface with the dimension of $436.8 \mathrm{~mm} \times 312 \mathrm{~mm}$ has been fabricated with LPKF ProtoLaser. It is composed by $14 \times 10$ lattices. Each lattice is a subarray composed by $12 \times 12$ " 0 " or " $\pi$ " elements. " 0 " and " $\pi$ " lattices are arranged periodically as shown in Figure 3(a). In this paper, full-wave simulation of the full structure was carried by CST Microwave Studio.

The monostatic RCS reduction was presented at Figure 4. The metasurface and the equal-sized PEC ground were measured at $x$-polarized wave normal incidence. The $-10 \mathrm{~dB}$ measured bandwidth is $89 \%$ (from 14.8 to $38.5 \mathrm{GHz}$ ) for the metasurface with periodic arrangement. The tendency of simulated result is similar to the measurements. The maximum RCS reduction has been obtained at $17 \mathrm{GHz}$ with a value around $-35 \mathrm{~dB}$. Note that the simulated bandwidth has been calculated by reflection coefficient. The achieved bandwidth is in good agreement with the predicted bandwidth based on the $180^{\circ}$ phase difference between two unit cells as shown in Figure 2.

The 3D scattering patterns of the periodic arrangement for normal incidence at $17 \mathrm{GHz}$ are presented in Figure 5. For the simulation result, the normalization value is $20.77 \mathrm{~dB}$. The EM wave scatters along four diverging directions. Hence, in the incident direction the scattered field was greatly suppressed, and monostatic RCS was effectively reduced. The analytical predictions are in good agreement with the fullwave simulations. Theoretical directions of four scattered beams can be found in $[6,8]$. The fixed number of scattered beams results in the fixed reduced magnitude of bistatic RCS.

\section{Optimized Phase Layouts}

From the foregoing, periodic arrangement produces four or six scattered beams and can greatly reduce the monostatic RCS [5-8]. However, bistatic RCS reduction is not very obvious and is -8.1 and $-8.9 \mathrm{~dB}$ for the rectangular and hexagonal surfaces, respectively. Optimizing the lattice layouts aims to redirect EM scattering to more directions and further reduce the bistatic RCS based on the energy conservation.

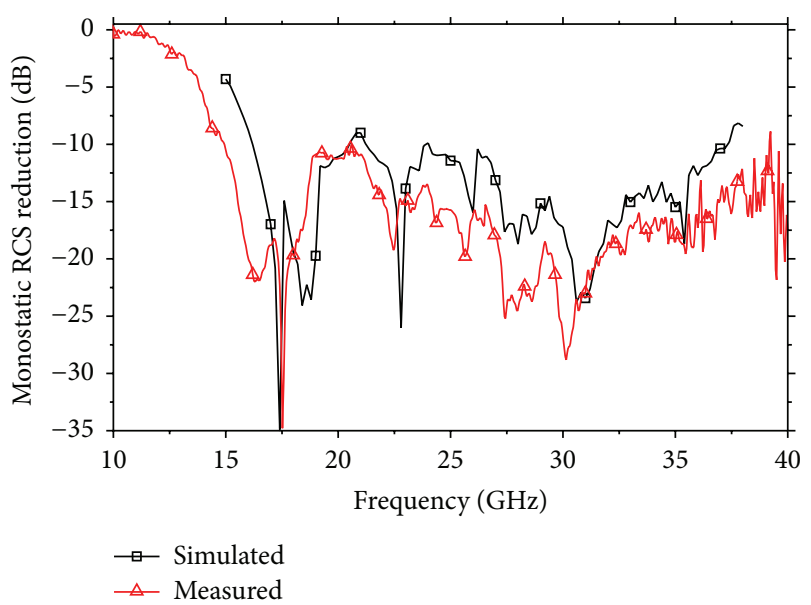

FIGURE 4: Simulated and measured results of monostatic RCS reduction for periodic arrangement at normal incidence.

We consider a rectangular metasurface with the same size as the metasurface of periodic phase arrangement that contains $14 \times 10$ equal-sized lattices in which each lattice is occupied by a subarray of " 0 " or " $\pi$ " elements. The distribution of " 0 " and " $\pi$ " lattices can be arbitrary. For simplicity, the planar array was considered to be the combination of two separable linear arrays; the optimized phase sequences can be designed independently along both the horizontal and vertical directions. The main aim of the study is to find a set of phase sequences in order to produce a scattering pattern with lowest possible value of maximum lobe level (MLL) and deep null placed in monostatic direction.

To get best performance of the metasurface, a particle swarm optimization (PSO) algorithm is utilized together with far field pattern prediction [17] to achieve the optimal phase arrangement. $d_{x}$ and $d_{y}$ are both set to $2 \lambda$. Accordingly, the periods of array factor are $P_{U}=P_{V}=0.5$. The RCS pattern of one lattice is shown in Figure 6. As electrical size of the lattice composed of $12 \times 12$ " 0 " or " $\pi$ " elements is relatively larger, in order to exactly model the scattering pattern of one lattice the $\mathrm{EF}$ is set to be 15. Fast Fourier transformation (FFT) is used to 


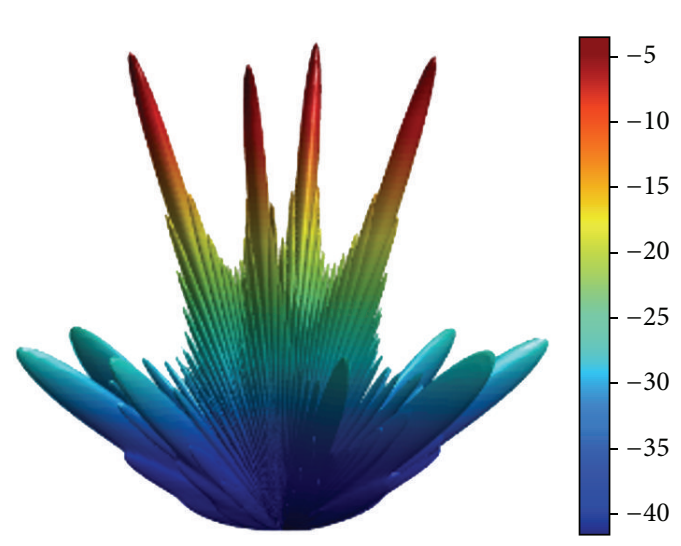

(a)

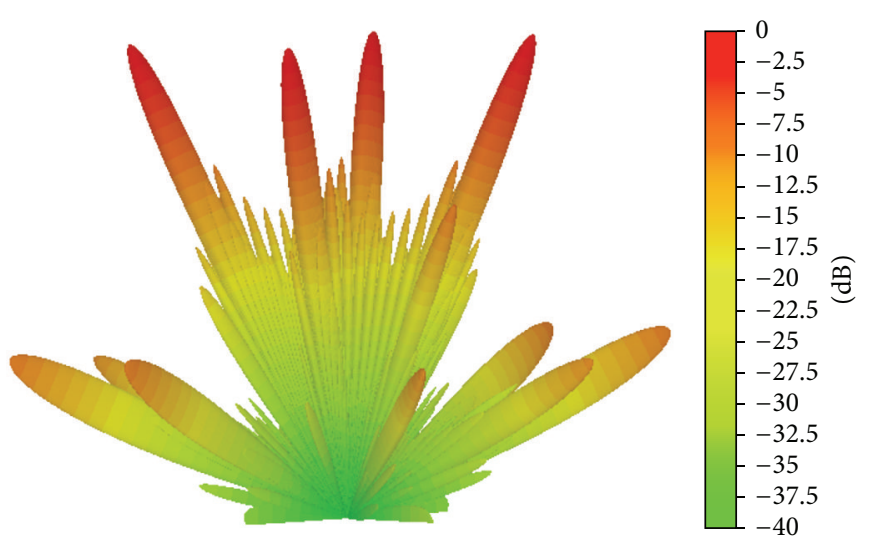

(b)

FIGURE 5: Scattering patterns of the metasurface with periodic arrangement at $17 \mathrm{GHz}$. (a) The normalized analytical results calculated by (3). (b) The full-wave simulation results.

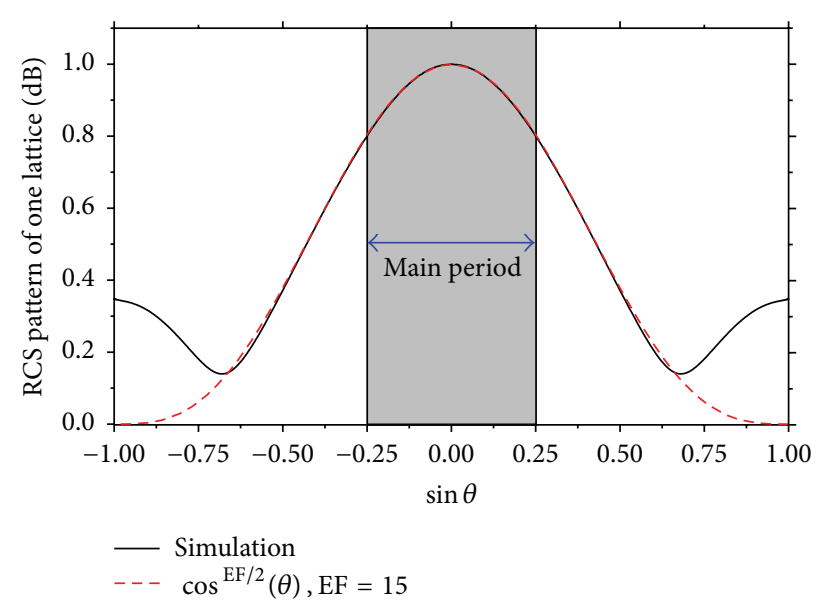

FIGURE 6: RCS pattern of one lattice composed by $12 \times 12$ " 0 " or " $\pi$ " elements.

accelerate the calculation of array factor. The score function is defined as

$$
\text { score }=\text { bistatic } \mathrm{RCS}+k \cdot(\text { monostatic } \mathrm{RCS}) \text {, }
$$

where $k$ is weighting factor that can balance the optimization results for both monostatic and bistatic. Through numerical test, we can find that the best optimization results can be obtained when $k=0.25$.

The optimized scattering patterns within the main period for lattice number of 14 and 10 are shown in Figure 8. Two corresponding phase sequences are " $0 \pi 00 \pi 0 \pi 0 \pi 0 \pi \pi \pi 0$ " and " $00 \pi \pi 0 \pi 0 \pi 0 \pi$ ", respectively. Figure 7 depicts the convergence curves of the PSO optimization. The flat scattering pattern means that scattered fields are suppressed in all directions, so that bistatic RCS can be effectively reduced. There is deep null at the direction $\theta=0$; thus the monostatic RCS can be greatly reduced. The main beam is suppressed and the EM energy is dispersed to the other direction. Our proposed physical mechanism follows the law of conservation of energy. As shown in Figure 8, the number of maximum

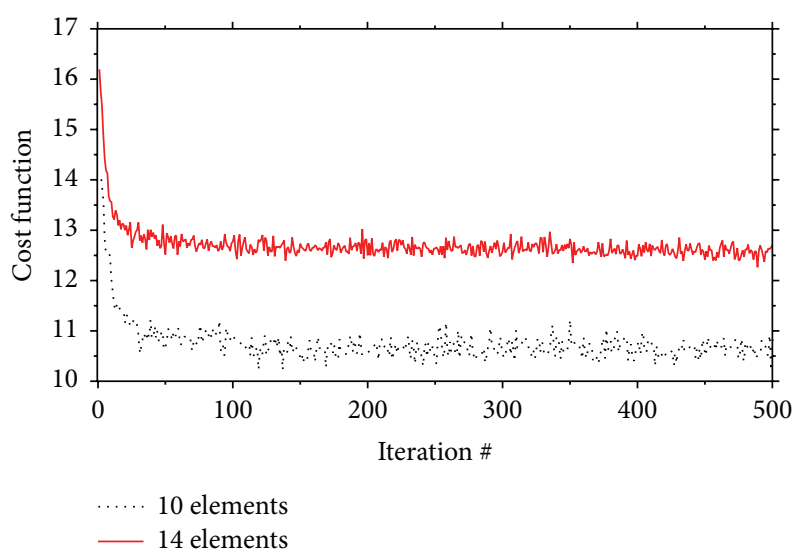

FIGURE 7: Convergence curves of the PSO optimization by using a 100 -agent swarm for 500 iterations and applying the cost function defined in (10).

points of the optimized results along the horizontal and vertical directions is 10 and 8 , respectively. Thus, in theory, the number of scattering beams is 80 within the main period. The scattering patterns are both symmetrical along the horizontal and vertical directions.

For a uniformly excited $N$-element array, the array factor pattern in one period has $N-1$ lobes including main beam, according to array theory. It means that a flat scattering pattern with more scattering beams can be synthesized by optimizing the phase arrangement as lattice number $(N)$ increases. Thus, as the size of the metasurface increases, the magnitude of bistatic RCS reduction becomes larger. It is worth mentioning that periodic phase arrangement produces a fixed number of scattering beams. Four and six fixed scattering beams are created for square and hexagonal $\mathrm{AMC} / \mathrm{EBG}$ surfaces [5-8], respectively. So the magnitude of maximum RCS reduction is also fixed and does not vary with the size changes.

To illustrate the above physical mechanism, we manufacture a metasurface based on two optimized phase sequences 


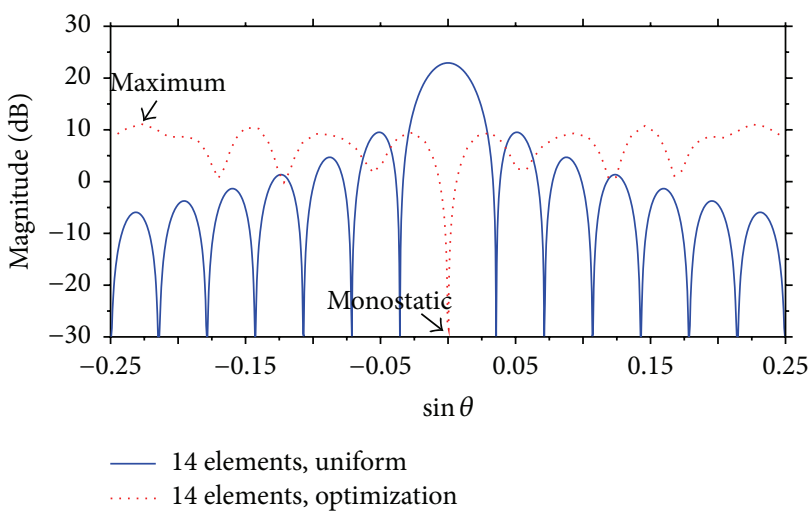

(a)

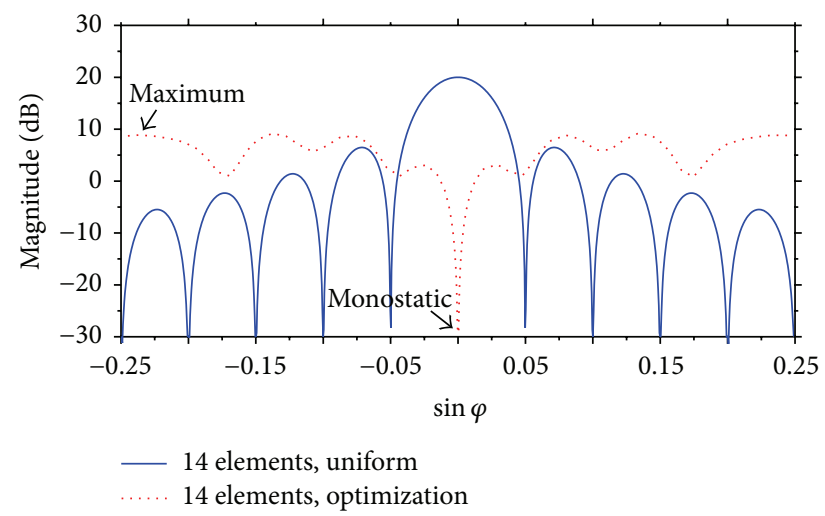

(b)

FIGURE 8: Far field scattering pattern along (a) horizontal direction and (b) vertical direction within the main period obtained by PSO.

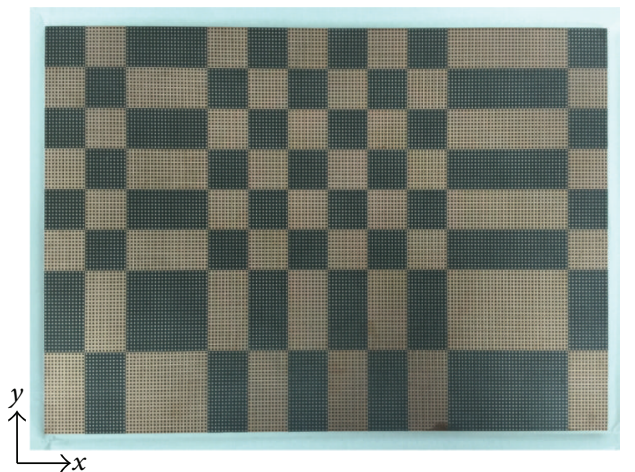

FIGURE 9: The manufactured metasurface based on two optimized phase sequences.

as shown in Figure 9. The simulated and measured results of monostatic and bistatic RCSs reductions under $x$-polarized and $y$-polarized normal incidences are presented in Figures 10 and 11, respectively. The same results of monostatic and bistatic RCSs reductions for $x$-polarization and $y$-polarization exhibit the polarization-independent feature of the metasurface. Due to the symmetric geometry of EBG unit cell, its phase responses are the same for both $x$-polarized and $y$-polarized waves; therefore metasurface is polarizationindependent. As shown, more than $80 \%$ frequency bandwidth with both monostatic and bistatic RCSs reductions larger than $9.5 \mathrm{~dB}$ is obtained by optimized phase layout. For monostatic RCS reduction shown in Figure 10, the measurements agree well with the simulated predictions. As shown in Figure 11, the bistatic RCS for periodic arrangement reduces at the level of about $-8.1 \mathrm{~dB}$, which is analogous to previous results reported in [7]. However, bistatic RCS can be further greatly reduced in broadband by optimized phase layout. Particularly at the frequency with exact $180^{\circ}$ phase difference, bistatic RCS reduction reaches $17.6 \mathrm{~dB}$.

The 3D RCS patterns of the metasurface for $x$-polarization at $17 \mathrm{GHz}$ are shown in Figures 12 and 13. Nearly the same results, which are not included here to minimize duplication, were obtained for $y$-polarization. As shown, scattering energy

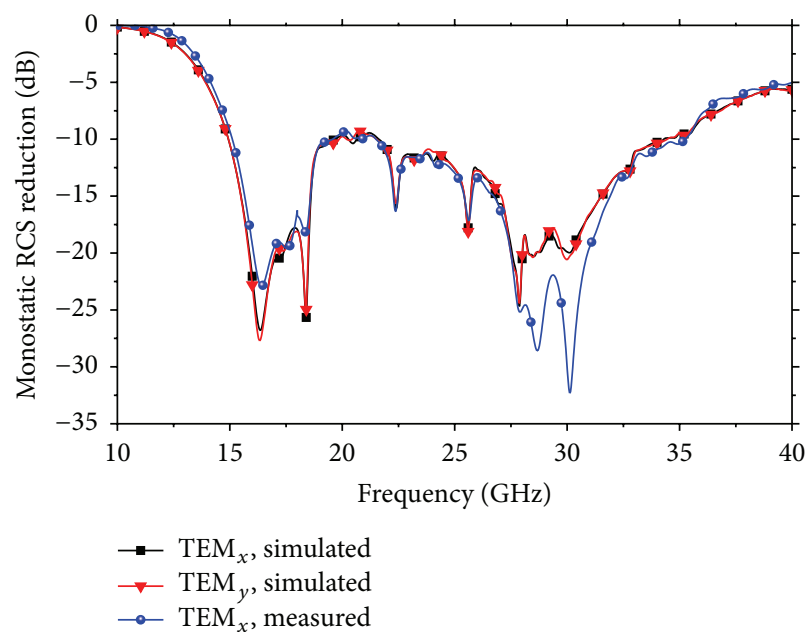

FIGURE 10: Simulated and measured results of monostatic RCS reduction for optimized phase layout at the normal incidence.

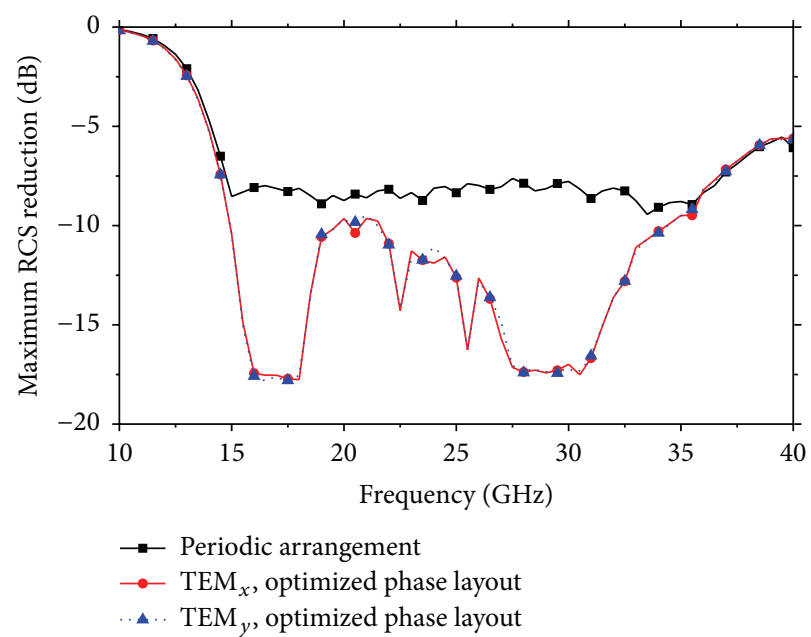

FIGURE 11: Simulated maximum RCS reduction for periodic arrangement and optimized phase layout. 


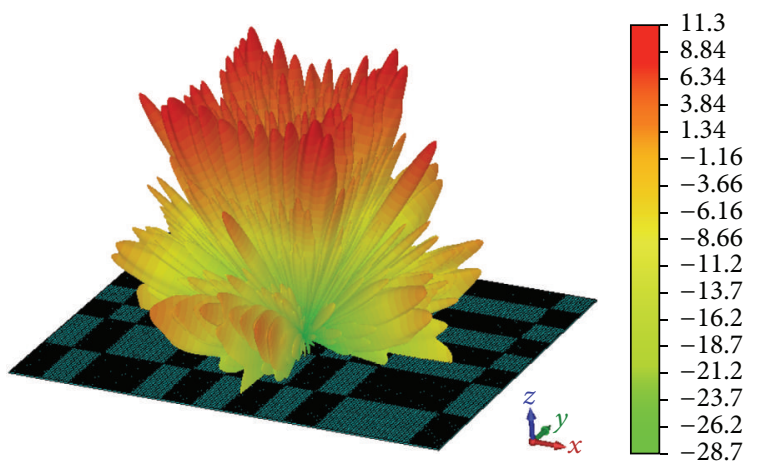

FIGURE 12: 3D bistatic RCS pattern for optimized phase layout at $17 \mathrm{GHz}$.

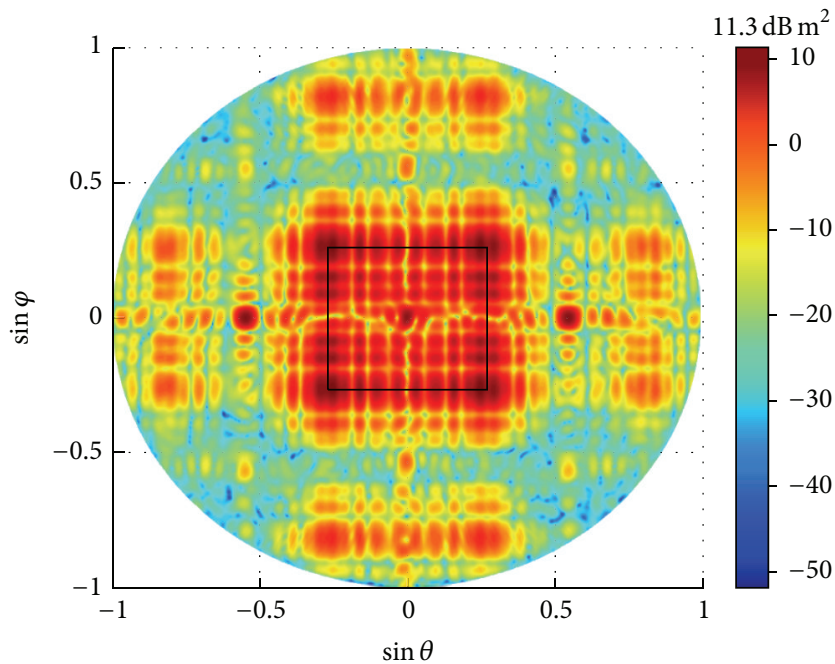

FIGURE 13: Contour plot of bistatic RCS pattern for optimized phase layout at $17 \mathrm{GHz}$. Black frame represents the main period.

focuses on the main period and its vicinity, because the scattering energy away from the main period is weakened by the lattice pattern. The scattered fields are suppressed in low levels in all directions, because the optimized phase layout has been designed to redirect the intercepted EM energies to more directions. A lot of bistatic RCS lobes are produced in space. For frequency point at $17 \mathrm{GHz}$, the lattice space $d_{x}=d_{y}=1.768 \lambda_{0}$. Accordingly, the periods of array factors $\mathrm{AF}_{U}$ and $\mathrm{AF}_{V}$ are $P_{U}=P_{V}=0.566$. As shown in Figure 13, the scattering pattern is symmetric about $x o z$ plane and yoz plane. The spatial distribution of scattering beams of the main period is consistent with far field pattern predictions shown in Figure 8. 80 scattering beams are created within the main period, so in the whole space more than 80 scattering beams are produced, leading to a great reduction of bistatic RCS. Under normal incidence, the RCS is dramatically reduced along the principal planes ( $x o z, y o z)$, as shown in Figures 14 and 15.

The RCS pattern in $\varphi=46.5^{\circ}$ and $133.5^{\circ}$ plane, where the maximum lobe appears at $17 \mathrm{GHz}$, is shown in Figure 16 . The maximum RCS of the metasurface is reduced by $17.6 \mathrm{~dB}$,

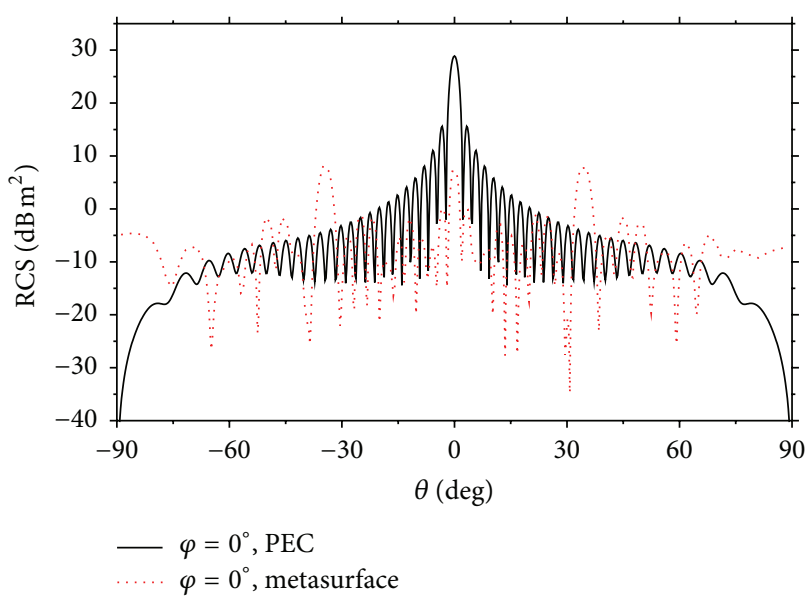

FIgURE 14: Comparison of the bistatic RCS at $17 \mathrm{GHz}$ along $x o z$ plane for the metasurface and the PEC plate.

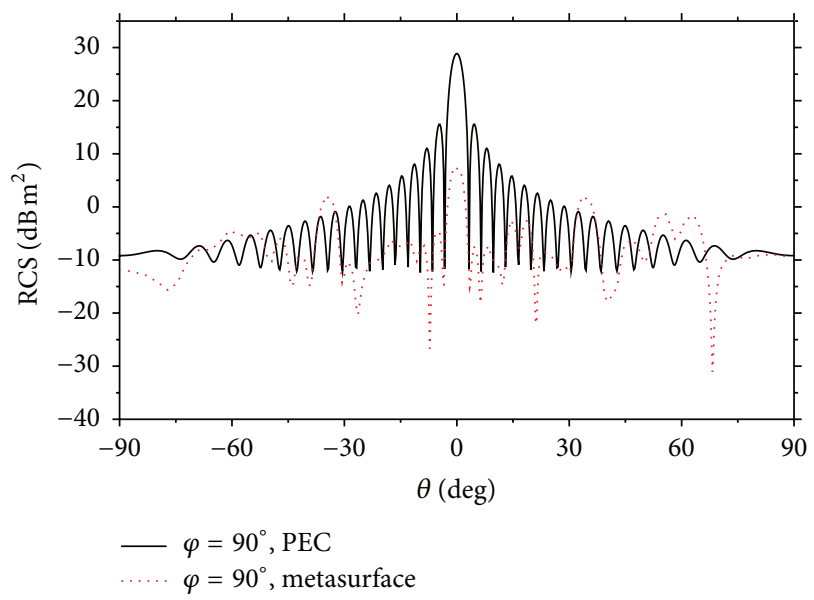

FIGURE 15: Comparison of the bistatic RCS at $17 \mathrm{GHz}$ along yoz plane for the metasurface and the PEC plate.

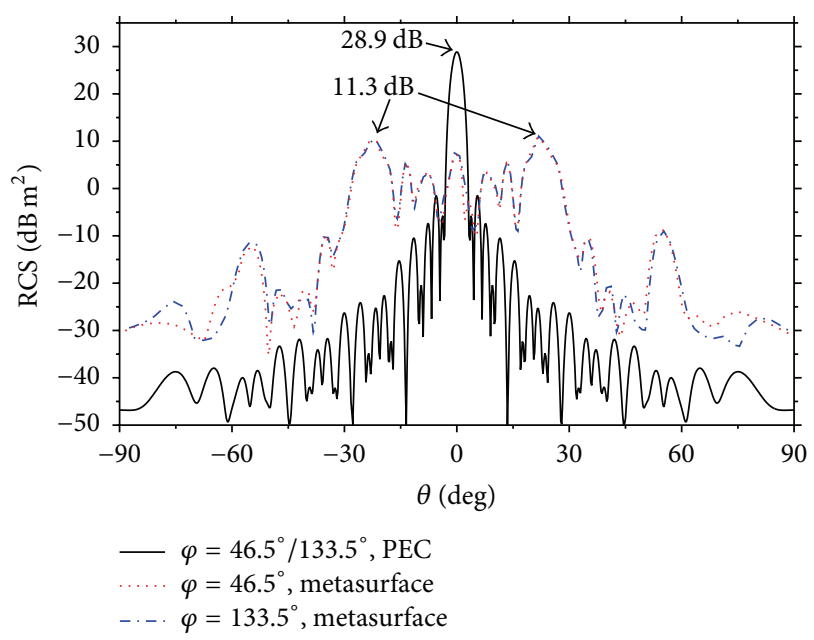

FIGURE 16: Comparison of the bistatic RCS at $17 \mathrm{GHz}$ along $\varphi=46.5^{\circ}$ and $133.5^{\circ}$ plane for the metasurface and the PEC plate. 
TABLE 1: Comparisons between the results of this work and earlier checkerboard surfaces.

\begin{tabular}{lcccc}
\hline & {$[6]$} & {$[7]$} & {$[8]$} & This work \\
\hline NSL & 4 & 4 & 6 & $>80$ \\
MRR $(\mathrm{dB})$ & -8.1 & -8.1 & -8.9 & -17.6 \\
BMRR & $40.2 \%(<-10 \mathrm{~dB})$ & $66.0 \%(<-6 \mathrm{~dB})$ & $63.0 \%(<-10 \mathrm{~dB})$ & $80 \%(<-9.5 \mathrm{~dB})$ \\
BBRR & - & $66.0 \%(<-6 \mathrm{~dB})$ & - & $80 \%(<-9.5 \mathrm{~dB})$ \\
\hline
\end{tabular}

NSL: the number of scattering lobes.

MRR: maximum RCS reduction.

BMRR: the bandwidth of monostatic RCS reduction.

BBRR: the bandwidth of bistatic RCS reduction.

which is much lower than that $(8.9 \mathrm{~dB})$ of the hexagonal EBG surface of periodic phase arrangement [8].

Scattering characteristics including the number of scattering beams, the magnitude of maximum RCS reduction, and the bandwidth of both monostatic and bistatic RCSs reductions, are compiled together in Table 1 under normal incidence. Comparing our results to recent research findings suggests that the proposed design approach has overwhelming advantages, especially in wideband suppression of bistatic RCS.

For the oblique incidence with different polarization, the RCS reductions in specular direction are given in Figure 17. Incident angle and polarization of plane wave result in the change of reflection phase difference between two unit cells. For TE polarization, as the incident angle increases, the operating frequency band shifts to the higher frequency. For TM polarization, as the incident angle increases, the high frequency band shifts to the higher frequency and the magnitude of specular RCS reduction becomes smaller at center frequency. The bistatic RCS patterns of the metasurface for oblique incidence with TE polarization at $17 \mathrm{GHz}$ are shown in Figure 18. The optimized phase arrangement has been designed to redirect the intercepted EM energies to more directions. The specular reflections will no longer dominate within the whole scattered waves.

\section{Conclusion}

A broadband ultrathin nonabsorptive metasurface is analyzed and designed for broadband monostatic and bistatic RCSs reductions in this paper. A new mechanism is proposed for manipulating EM scattering and realizing the best reduction of monostatic and bistatic RCSs through controlling reflection phase of EM wave impinging on metasurface. The bandwidth of more than $80 \%$ is achieved at the normal incidence for the $-9.5 \mathrm{~dB}$ RCS reduction for both monostatic and bistatic. The experiment results are in good agreement with the corresponding simulations. Experimental and simulated results validate the effectiveness of our approach.

Periodic Arrangement. Because the scattered fields are redirected toward four and six directions for the rectangular and hexagonal [5-8] checkerboard surfaces, respectively, the maxima of the bistatic RCS are -8.1 and $-8.9 \mathrm{~dB}$ compared to those of the equal-sized PEC ground planes.

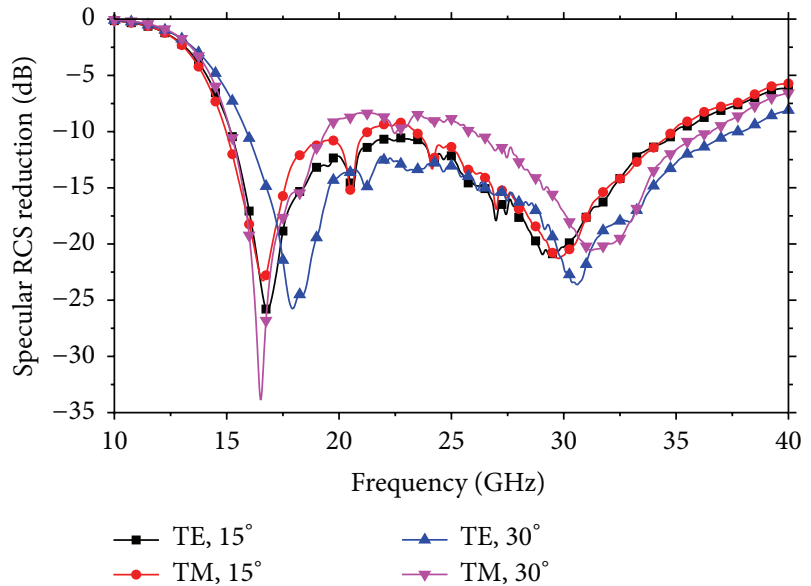

FIGURE 17: Simulated RCS reduction in specular direction for oblique incidence.

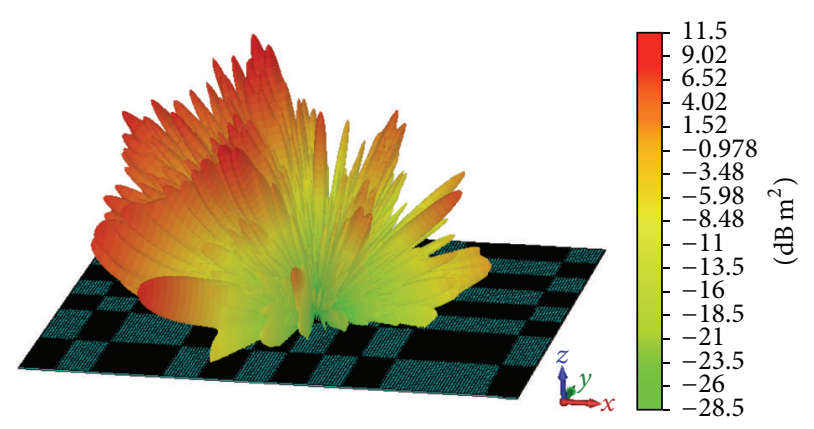

FIGURE 18: 3D plot of the bistatic RCS pattern at $17 \mathrm{GHz}$ for $\left(\theta^{\text {inc }}=\right.$ $\left.30^{\circ}, \mathrm{TE}\right)$.

Optimized Phase Layout. The intercepted EM energies were redirected to more directions, so that the scattered fields are controlled in low levels in all directions. A lot of bistatic RCS lobes ( $\gg 4$ or 6 ) are produced in space, leading to a great bistatic RCS reduction. Furthermore, the number of RCS lobes increases as metasurface size (lattice number) increases; thus the reduced magnitude of monostatic and bistatic RCSs becomes greater. For the metasurface designed based on optimized phase layout in this paper, more than 80 scattering beams are created in space, and the bistatic RCS reduction reaches $17.6 \mathrm{~dB}$. 


\section{Competing Interests}

The authors declare that there are no competing interests regarding the publication of this paper.

\section{Acknowledgments}

The work was supported in part by the National Science Foundation of China (NSFC) under Grant nos. 61331002, 61102011, and 61201082 and in part by Excellent Innovation Team of CUC under Grant no. yxtd201303.

\section{References}

[1] M. A. Kats, D. Sharma, J. Lin et al., "Ultra-thin perfect absorber employing a tunable phase change material," Applied Physics Letters, vol. 101, no. 22, Article ID 221101, 2012.

[2] M. Aldrigo, M. Dragoman, A. Constanzo, and D. Dragoman, "Graphene as a high impedance surface for ultra-wideband electromagnetic waves," Journal of Applied Physics, vol. 114, no. 18, Article ID 184308, 2013.

[3] H. H. Tran and I. Park, "Wideband circularly polarized lowprofile antenna using artificial magnetic conductor," Journal of Electromagnetic Waves and Applications, vol. 30, no. 7, pp. 889897, 2016.

[4] M. M. Tahseen and A. A. Kishk, "Ka-band circularly polarized high efficiency wide band reflectarray using cross bow-tie elements," Progress in Electromagnetics Research, vol. 153, pp. 110, 2015.

[5] M. Paquay, J.-C. Iriarte, I. Ederra, R. Gonzalo, and P. de Maagt, "Thin AMC structure for radar cross-section reduction," IEEE Transactions on Antennas and Propagation, vol. 55, no. 12, pp. 3630-3638, 2007.

[6] Y. Zhang, R. Mittra, B.-Z. Wang, and N.-T. Huang, "AMCs for ultra-thin and broadband RAM design," Electronics Letters, vol. 45, no. 10, pp. 484-485, 2009.

[7] Y. Li, J. Zhang, S. Qu et al., "Wideband radar cross section reduction using two-dimensional phase gradient metasurfaces," Applied Physics Letters, vol. 104, no. 22, Article ID 221110, 2014.

[8] W. Chen, C. A. Balanis, and C. R. Birtcher, "Checkerboard EBG surfaces for wideband radar cross section reduction," IEEE Transactions on Antennas and Propagation, vol. 63, no. 6, pp. 2636-2645, 2015.

[9] X. M. Yang, G. L. Jiang, X. G. Liu, and C. X. Weng, "Suppression of specular reflections by metasurface with engineered nonuniform distribution of reflection phase," International Journal of Antennas and Propagation, vol. 2015, Article ID 560403, 8 pages, 2015.

[10] S. J. Li, J. Gao, X. Y. Cao, W. Q. Li, Z. Zhang, and D. Zhang, "Wideband, thin, and polarization-insensitive perfect absorber based the double octagonal rings metamaterials and lumped resistances," Journal of Applied Physics, vol. 116, no. 4, Article ID 043710, 2014.

[11] H. Xiong, L.-L. Zhong, C.-M. Luo, and J.-S. Hong, "Dualband polarization-/angle-insensitive metamaterial absorber," AIP Advances, vol. 5, no. 6, Article ID 067162, 2015.

[12] P. K. Singh, S. K. Ameri, L. Chao, M. N. Afsar, and S. Sonkusale, "Broadband millimeterwave metamaterial absorber based on embedding of dual resonators," Progress in Electromagnetics Research, vol. 142, pp. 625-638, 2013.
[13] M. S. Rill, C. Plet, M. Thiel et al., "Photonic metamaterials by direct laser writing and silver chemical vapour deposition," Nature Materials, vol. 7, no. 7, pp. 543-546, 2008.

[14] N. Yu, P. Genevet, M. A. Kats et al., "Light propagation with phase discontinuities: generalized laws of reflection and refraction," Science, vol. 334, no. 6054, pp. 333-337, 2011.

[15] C. A. Balanis, Antenna Theory: Analysis and Design, John Wiley \& Sons, New York, NY, USA, 3rd edition, 2005.

[16] E. F. Knott, Radar Cross Section Measurements, Springer, Berlin, Germany, 2012.

[17] C. Han and L. Wang, "Array pattern synthesis using particle swarm optimization with dynamic inertia weight," International Journal of Antennas and Propagation, vol. 2016, Article ID 1829458, 7 pages, 2016. 


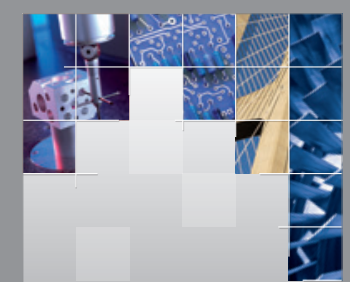

\section{Enfincering}
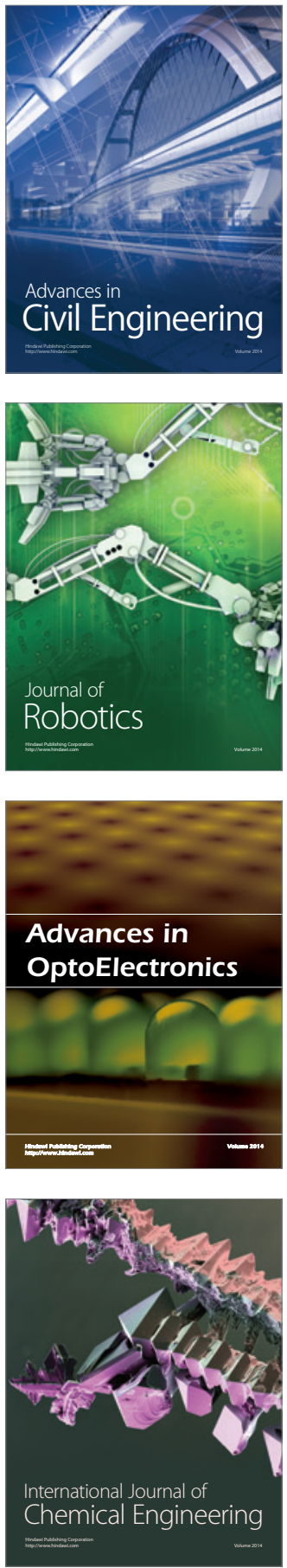

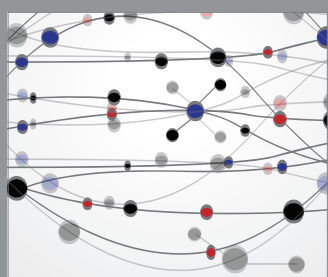

The Scientific World Journal

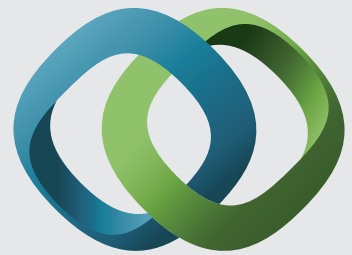

\section{Hindawi}

Submit your manuscripts at

http://www.hindawi.com
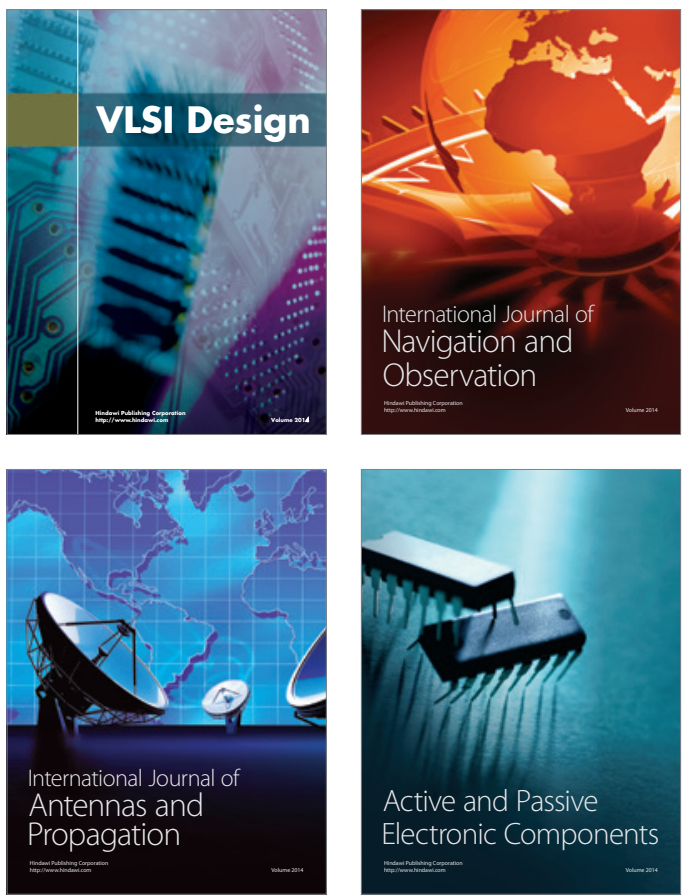
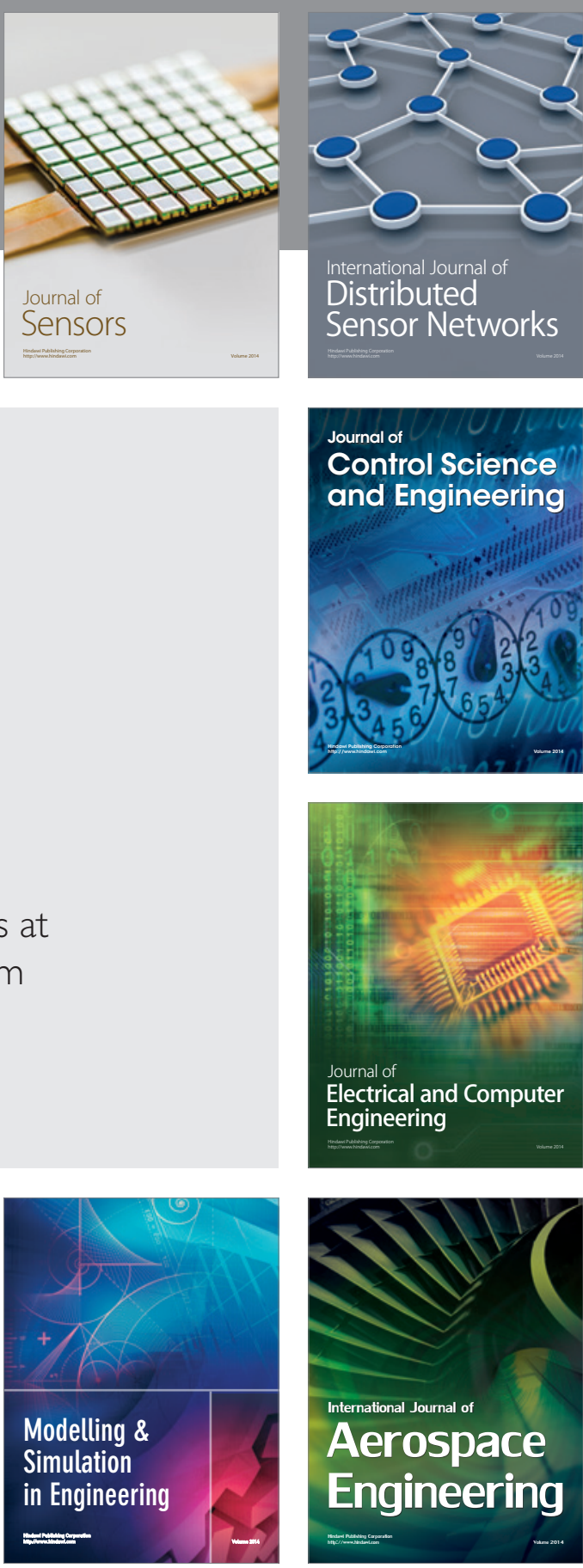

International Journal of

Distributed

Sensor Networks

Journal of

Control Science

and Engineering
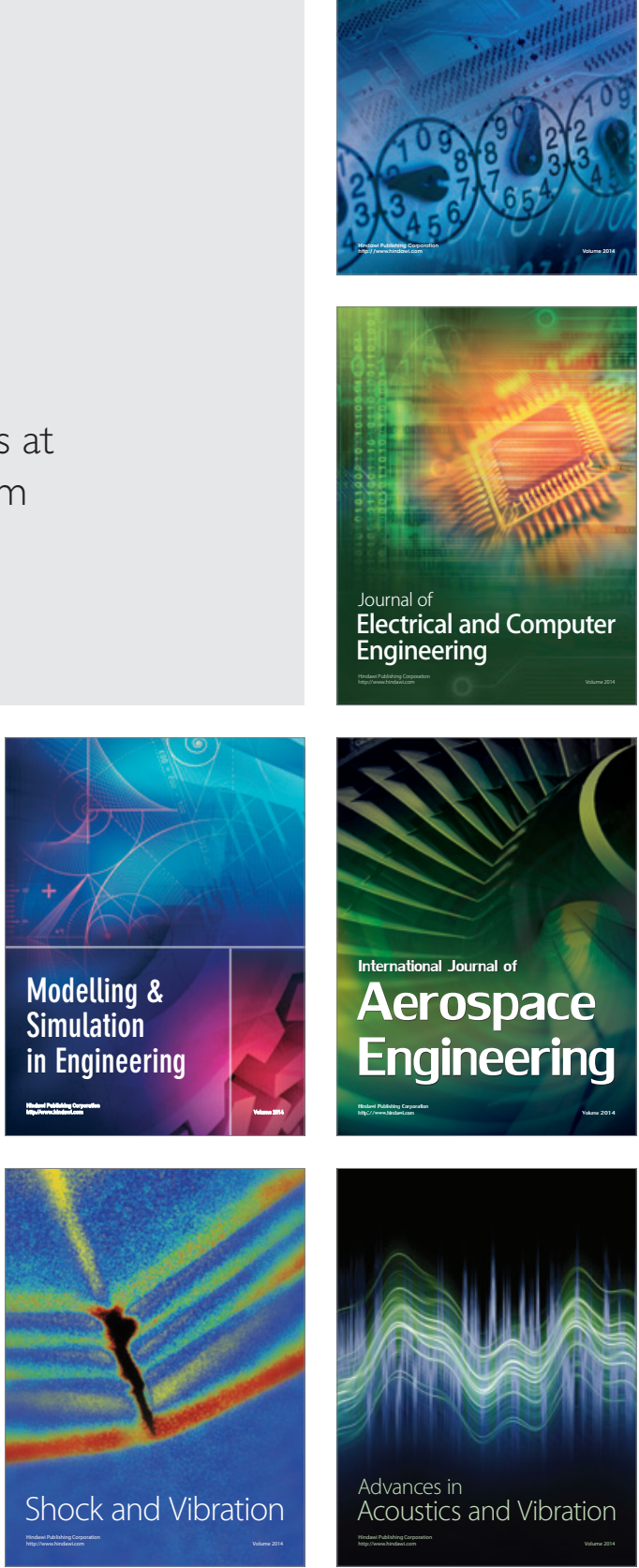